\title{
Modelling Groundwater Dependent Ecosystems in the Willunga Basin, South Australia
}

\author{
S.H. Hamilton ${ }^{\text {a }}$, J.H.A. Guillaume ${ }^{a}$ and S. EISawah ${ }^{a}$ \\ ${ }^{a}$ National Centre for Groundwater Research and Training (NCGRT), The Fenner School of Environment and \\ Society, The Australian National University, Australian Capital Territory \\ Email: serena.hamilton@anu.edu.au
}

\begin{abstract}
The challenge of groundwater management is to establish sustainable extraction regimes that provide acceptable levels of protection of economic, social and environmental assets and values that depend on the resource. A key knowledge gap relates to how groundwater resource management affects the integrity and survival of groundwater dependent ecosystems (GDEs). This study involves the development of habitat suitability models that assess the suitability of the groundwater regime in the Willunga Basin for supporting GDEs. GDE species in the Basin are classified into five functional groups according to their water requirements and tolerances. Habitat suitability index curves were developed for the five groups based on species observations and descriptions reported in literature. The index curves are described by a set of constraints that quantify the minimum and maximum bounds of values, reflecting the uncertainty of the relationship between groundwater regime and species habitat suitability. Alternative mathematical translations of descriptions of the groundwater requirements of species were also tested. The models were applied to assess the habitat suitability of the five functional groups at 35 sites in the Willunga Basin in terms of the groundwater regime (results are summarised in the figure below).
\end{abstract}
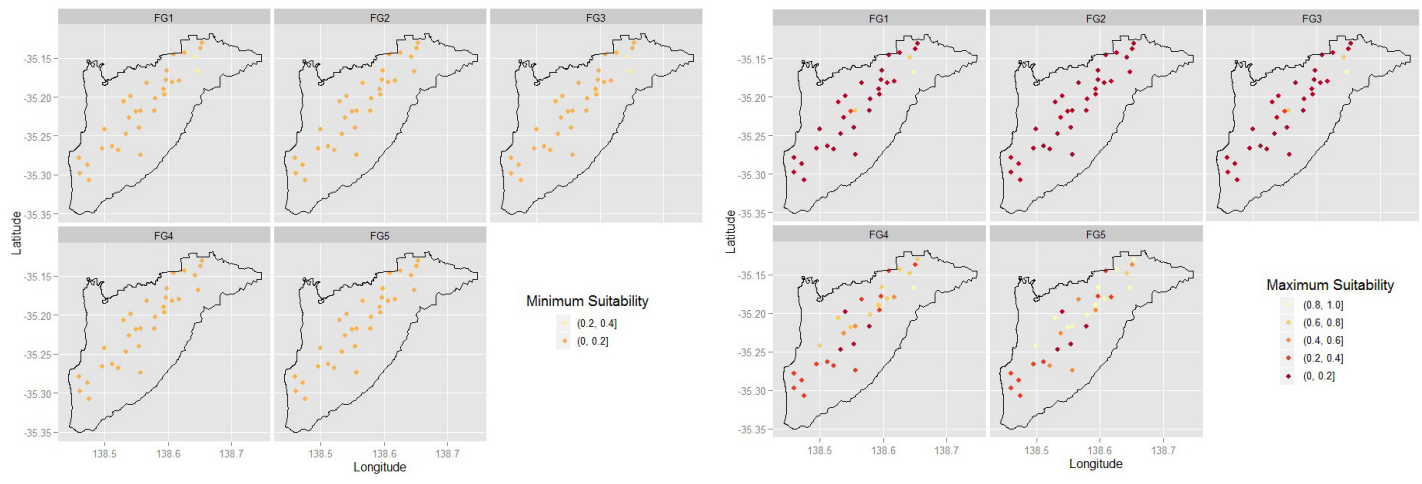

Figure 1. The minimum (left) and maximum (right) habitat suitability values for the five functional groups of plant assemblages across 35 sites in the Willunga Basin.

The models were able to identify sites that have unsuitable or poorly suited habitat for most of the functional groups with high confidence. However, there was low confidence in identifying sites with good habitat. The results of test runs of the alternative constraints suggested that the models were relatively robust. The sites were ranked by partial order, however if the plausible index bounds of sites overlapped, uncertainty remained about the ranking implied. Large bounds indicate gaps in knowledge that require further research. The model was applied to detect areas where GDE habitat may have been lost through time. Potential applications of the model include identifying sites for further monitoring or research. Future work should include the incorporation of more attributes of the groundwater regime, water quality and other environmental factors to describe habitat suitability, the use of ecological data for calibrating or validating the model, and the involvement of experts to set and review the model constraints. This modelling approach allows sites to be evaluated from an ecological point of view even with high uncertainty.

Keywords: Ecological modelling, groundwater dependent ecosystems, habitat suitability model, uncertainty 


\section{INTRODUCTION}

Competition for water resources has intensified from increasing agricultural, industrial and domestic demand. Consequently, the importance of closing the gaps in our knowledge of the environmental impact of water resource development has increased. One key area that remains poorly understood relates to groundwater processes and the ecosystems that depend on this unseen yet vital resource (Mackay, 2006). To determine sustainable groundwater extraction regimes, it is crucial that we understand what factors influence the ability of an area to support groundwater-dependent ecosystems (GDEs). This study will examine GDEs in the Willunga Basin, a multi-aquifer groundwater system in South Australia. The study is part of a wider project that is examining the social, economic, ecological and policy dimensions of groundwater resource management, through the development of integrated models and decision support tools that characterise tradeoffs between these dimensions (El Sawah et al., 2011).

In March 2012, we ran a workshop to bring together a number of experts on GDEs (i.e. from CSIRO, NRM board, Department of Water, NCGRT, SKM) to discuss the design and implementation of the ecological component. We shared with experts the alternative approaches for GDE modelling and sought their feedback on the best approach to be used for the Willunga Basin in terms of the system's nature, data availability and stakeholder's interest. Experts provided useful feedback that was used to guide the modelling reported in this document. This includes:

1. Considering functional groups (rather than individual species) with the same groundwater requirements. Functional groups, as defined in the EA and SKM (2012) report, were recommended as a good starting point.

2. Developing a conceptual model to incorporate all processes and drivers (not only groundwater) as well as interacting ecological functions.

\section{Consider the suitability of sites for each of the functional groups}

Uncertainties about the specific groundwater requirements of GDEs in the Basin are high, not only due to the limited field data on GDEs in the Basin but also the complexities of ecological dependence on groundwater in general. The groundwater requirements of a GDE assemblage or species can vary in each situation subject to other conditions. For example, dependence on groundwater will be greater during drought periods, when access to other water supplies (surface water or rainfall) is diminished. Another major factor that confounds understanding of the groundwater requirements of GDEs is the varying degree of dependency on the water resource; for some GDEs, continuous access to groundwater is essential for survival, whereas for others the role of groundwater is minor or non-essential.

The high uncertainty about the habitat requirements of GDEs prevents the transference of the available, albeit limited, ecological knowledge to most types of models. This study applies an index-based habitat suitability modeling approach, developed in $\mathrm{Fu}$ and Guillaume (under review) that captures uncertain knowledge by stating parameter values in terms of plausible bounds. Habitat suitability index curves are developed to describe the minimum and maximum bounds of the relationship between groundwater regime and habitat suitability for five functional species groups. The aim of this study is to assess the suitability of the groundwater regime of sites in the Willunga Basin for supporting GDEs under uncertainty. These models are applied to compare and partially rank 35 sites according to their suitability to support different GDEs and to identify sites that appear to have undergone major change in their ability to support GDEs and warrant further investigation. This study also assesses the impact of alternative parameter values on model outputs.

\section{STUDY AREA}

The Willunga Basin is located approximately $35 \mathrm{~km}$ south of Adelaide, South Australia. The Basin contains four aquifer systems: the Maslin Sands, Port Willunga Formation, Fractured Rock (Basement) and Quaternary aquifers (Figure 2). Maslin Sands and Port Willunga Formation aquifers supply about $85 \%$ of groundwater pumped in the Basin for irrigation (Department for Water, 2012). Groundwater tends to flow from the northeast corner of the Basin towards the coast. Maslin Sands and Port Willunga are sedimentary aquifers, which depend on recharge from 1) direct rainfall infiltration, 2) streams and 3) outflow from the basement rocks. Recharge via direct rainfall infiltration only occurs where the aquifers outcrop at or

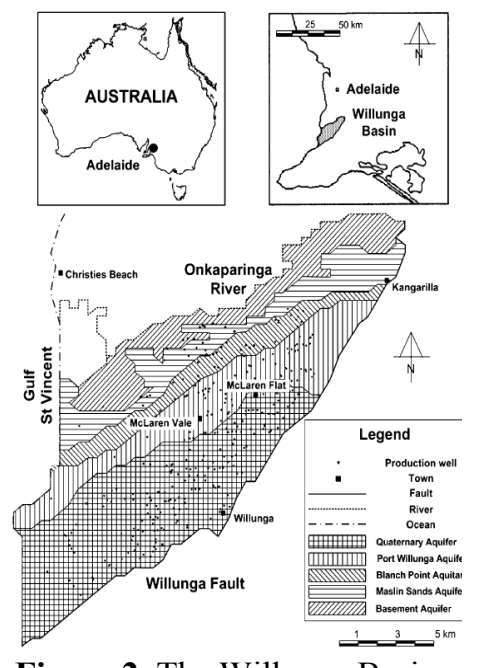

Figure 2. The Willunga Basin (source: Knowles et al., 2007) 
very close to the surface. Most aquifers are confined over most of the Basin, so there is usually no direct correlation between rainfall and groundwater level fluctuations (Department for Water, 2012). In addition to extraction, groundwater outflow also occurs from evapotranspiration, and discharge to the sea, wells and streams.

Willunga Basin is a high agricultural production area, with wine-grape production its primary industry. Since the 1970s, both the level and quality of groundwater (especially in terms of salinity) in the Basin has declined, primarily due to growth in the viticulture industry, which relies mostly on groundwater irrigation (Knowles et al., 2007). Due to the decline in groundwater levels, in 1999 the local water authority significantly reduced the extraction rates allowed by users. Despite this reduction in allowable extraction rates, groundwater levels continued to decline over the period of 2001 to 2006, which experienced below average rainfall (Knowles et al., 2007). Groundwater levels have stabilised since 2006, however salinity levels have continued to rise across the Basin (Department of Water, 2012). In the early to mid-20 century, extensive drainage systems were constructed in parts of the Basin to reclaim flooded land for agricultural development and to prevent flooding of assets (EA 2003). Numerous dams and other water resource development structures were also constructed throughout the Basin (AMLRNRM, 2007). These development works significantly altered the nature of the groundwater systems, however as groundwater monitoring only begun in the late $20^{\text {th }}$ century the extent of change remains unknown (EA 2003).

\section{GROUNDWATER DEPENDENT ECOSYSTEMS}

There are three main types of GDEs, those dependent on surface expression of groundwater (e.g. discharge into a wetland or river), those dependent on subsurface expression of groundwater (e.g. below the surface but within the rooting depth), and those that reside in the aquifer itself. This study focuses on plant communities that depend on the subsurface expression of groundwater. Some plant communities may utilize a combination of groundwater accessed through subsurface and surface expression, however at this stage of the project we will not consider the ecological requirements associated with the complex interplay between subsurface and surface water processes due to the absence of information on this.

This study recognises five functional groups of plant assemblages in the Willunga Basin, as described in EA and SKM (2012). The first three functional groups occur in perennially saturated soils. Functional Group 1 (FG1) occur in groundwater seep areas with no or shallow flooding (drainage features absent), and its species generally cannot tolerate flow. FG2 occur along watercourses where groundwater discharges, and its species are adapted to flow conditions. FG3 occur in swamp and marsh areas with weak flow, where flooding can persist for several months. FG4 is found in sites that are seasonally waterlogged, but dry out in summer and autumn. FG5 assemblages are intolerant of waterlogging, occurring in sites with well-drained soils overlying a shallow aquifer (EA and SKM 2012). Table 1 summarises the requirements of these five groups and includes examples of plant species in the Willunga Basin in each group. It should be noted that species, for example river red gum and red-fruit saw-sedge, can fall in multiple categories.

Table 1: The water requirements of the five functional groups (EA and SKM, 2012)

\begin{tabular}{|l|l|l|l|}
\hline Group & $\begin{array}{l}\text { Saturation } \\
\text { conditions }\end{array}$ & $\begin{array}{l}\text { Flow and flooding } \\
\text { tolerance }\end{array}$ & Plant species \\
\hline FG1 & $\begin{array}{l}\text { Permanent } \\
\text { waterlogging }\end{array}$ & $\begin{array}{l}\text { Intolerant of strong } \\
\text { flow; No or shallow } \\
(<0.2 \mathrm{~m} \text { ) flooding }\end{array}$ & $\begin{array}{l}\text { Square twig sedge (Baumea tetragona), Slender twig sedge (Baumea } \\
\text { gunnii), Scrambling coral fern (Gleichenia microphylla), King fern } \\
\text { (Todea Barbara), Red-fruit saw-sedge (Gahnia sieberiana), Soft water- } \\
\text { fern (Blechnum minus) }\end{array}$ \\
\hline FG2 & $\begin{array}{l}\text { Permanent } \\
\text { waterlogging }\end{array}$ & $\begin{array}{l}\text { Tolerates flow; } \\
\text { Seasonal flooding } \\
\text { related to } \\
\text { streamflow }\end{array}$ & $\begin{array}{l}\text { Wirilda (Acacia provincialis), Tall sedge (Carex appressa), Leafy } \\
\text { twigrush (Cladium procerum), Tassel sedge (Carex fascicularis), Ruddy } \\
\text { ground fern (Hypolepis rugulosa), Shrubby fireweed (Senecio minimus) }\end{array}$ \\
\hline FG3 & $\begin{array}{l}\text { Permanent } \\
\text { waterlogging }\end{array}$ & $\begin{array}{l}\text { Tolerates weak or } \\
\text { low flow; Seasonal } \\
\text { shallow (<0.5 m) } \\
\text { flooding }\end{array}$ & $\begin{array}{l}\text { Common reed (Phragmites australis), Red-fruit saw-sedge, Woolly tea } \\
\text { tree (Leptospermum lanigerum), Prickly tea tree (Leptospermum } \\
\text { continentale), Square twig sedge }\end{array}$ \\
\hline FG4 & $\begin{array}{l}\text { Alternately } \\
\text { waterlogged and } \\
\text { drained soils }\end{array}$ & $\begin{array}{l}\text { Intolerant of flow; } \\
\text { Flooding rare or } \\
\text { absent }\end{array}$ & $\begin{array}{l}\text { Blackwood (Acacia melanoxylon), Bracken fern (Pteridium esculentum), } \\
\text { River red gum (Eucalyptus camaldulensis), Rush sedge (Carex } \\
\text { tereticaulis), Black bristlerush (Chorizandra enodis), Spiny flat-sedge } \\
\text { (Cyperus gymnocaulos), Variable saw sedge (Lepidosperma laterale) }\end{array}$ \\
\hline FG5 & $\begin{array}{l}\text { No waterlogging; } \\
\text { shallow watertable } \\
\text { below drained soils }\end{array}$ & Intolerant of flow & $\begin{array}{l}\text { River red gum, Pink gum (Eucalyptus fasciculosa) } \\
\end{array}$
\end{tabular}




\section{HABITAT SUITABILITY INDEX MODEL}

Habitat suitability index models use a numerical index to describe the potential capacity of a habitat in supporting a species or community, ranging from 1.0 (optimal habitat) to 0.0 (unsuitable habitat). These models incorporate hypotheses of relationships between the species and key habitat variables that may affect their survival, growth, abundance, distribution or health. These relationships are often expressed in the form of univariate curves or simple equations, derived from field or laboratory observations, published literature or expert judgment. A composite habitat suitability score is calculated by combining the variables, such that important or limiting variables are given more weight (Brooks, 1997). Habitat suitability index models are relatively simple ecological models that generally do not consider factors such as population dynamics, competition or predator-prey interactions, or historical disturbances. They also do not require species occurrence or abundance data to be developed; although such data would certainly increase the model's reliability and validity. Habitat suitability models are therefore much less data-intensive than other ecological models, and appropriate where knowledge and data are limited (Smith et al., 2007). The models in this study are yet to be validated; this is an important area for future work. Model validation involves testing the hypothesis that habitat suitability (i.e. the model output) correlates to species occurrence, and requires field data (e.g. species presence/absence or density) collected from the same site as the groundwater data.

\subsection{Data}

For this study, habitat suitability of each of the species functional groups is defined in terms of one attribute, depth to groundwater (SWL, standing water level). This groundwater data was accessed from the web-based data repository WaterConnect (https://www.waterconnect.sa.gov.au/Systems/GD/). The model test sites were limited to well locations in the Willunga Basin. SWL observations were collected from most wells on a monthly basis. Groundwater dependence is unlikely where water tables are more than $20 \mathrm{~m}$ (EA and SKM 2012), therefore sites with average SWL observations of more than $20 \mathrm{~m}$ were not considered. Sites with smaller data sets ( $<200$ data points) were also excluded from this study, leaving 35 sites (with observations spanning more than one decade) for further analysis.

\subsection{Habitat suitability index curves}

The approach proposed by $\mathrm{Fu}$ and Guillaume (under review) was applied, whereby the index curves are defined by the modeller in terms of plausible bounds, rather than precise best estimates. In their study, Fu and Guillaume (under review) used the approach to compare the suitability of surface water and groundwater regimes for three riparian plant species before and after a given point in time. In this study, the approach is applied to compare the suitability of groundwater levels for five functional species groups across a number of sites, and for each site over time. For each functional group, the modeller specifies a set of constraints in terms of the bounds (i.e. maximum and minimum values) of habitat suitability of attribute values (i.e. groundwater levels), the comparative suitability of attribute values (e.g. $<0.5 \mathrm{~m}$ is more suitable than $1.0-2.0$ $\mathrm{m}$ ) and the monotonicity (direction) of change in suitability. Qualitative descriptions of species that belonged to (or were assumed to belong to) each of the functional groups in relation to their groundwater level requirements or tolerances were translated into quantitative mathematical constraints. These habitat suitability constraints were developed through a review of literature.

The habitat suitability index is on a continuous scale, but can be interpreted as follows (McMahon 1983): 0.8 to $1.0=$ excellent habitat (corresponds to highest survival, growth and reproduction); 0.5 to $0.7=$ good habitat; 0.2 to 0.4 = fair habitat; and 0.0 to 0.1 = poor habitat (corresponds to high mortality and unsuccessful reproduction, approaching lethal conditions). Values in between these bands can be interpreted as intermediate classes, for example, $0.75=$ fair to good habitat.

FG1, FG2 and FG3 require permanent waterlogging, so these three groups are assumed to have the same groundwater level suitability constraints. These species groups differ in their occurrence along watercourse, which were defined as sites that occurred within a $20 \mathrm{~m}$ buffer of mapped watercourse lines. The $20 \mathrm{~m}$ figure was arbitrarily chosen; other distances were also tested in the study. FG1 does not occur along watercourses, therefore sites within the buffer zone of watercourse lines were given a habitat suitability rating of zero. Conversely, FG2 must occur along watercourses, and therefore only sites within the buffer zone were considered to have potentially suitable habitat. FG3 can occur near watercourses, but are not limited to such sites, and therefore no watercourse distance constraints were defined for this group, nor FG4 and FG5.

FG5 requires drained soils over a shallow water table; their habitat suitability constraints were based on observations of FG5 species and terrestrial GDE species. FG4 need groundwater levels that meet its species' wetting and drying cycles. The group requires seasonal waterlogging, and therefore the same groundwater 
suitability constraints as FG1, 2 and 3 are assumed for part of the year; these constraints are applied to the minimum SWL in each year instead of over all data points. FG4 also require drained soils for at least part of the year. To account for this requirement, the suitability constraints of FG5 are to be applied to the maximum SWL of each year. The index values calculated for the wetting and drying cycle are combined to give a composite habitat suitability index score for FG4, assuming the weight of each attribute is approximately the same (0.4-0.6/0.4-0.6).

The habitat suitability index models were posed as optimisation problems in the form of a set of linear programming problems. They were solved using the lpSolveAPI package in R. In recognition that the qualitative descriptions could have different quantitative translations, possible alternative mathematical translations were also tested. Although the 'best' estimates of plausible bounds are intended to capture uncertainty, these uncertainty estimates are themselves subject to uncertainty. These alternative translations were run, and their impact on the final results examined.

\section{RESULTS}

The model outputs included the minimum and maximum habitat suitability values for the five functional groups across all 35 sites, averaged over the years; these values are mapped out in Figure 1. These suitability bounds are also plotted in Figure 3, where the sites are sorted by the average of their minimum and maximum scores. This ranking is considered to only be of partial order due to the uncertainty of values as indicated by the plausible bounds. If the index bounds of sites overlapped, there is low confidence in the ranking implied in the plots. Given the model assumptions and constraints, none of the 35 sites tested were rated as having good or excellent habitat suitability for any of the functional groups with high confidence. On the other hand, more than half the sites were rated as having unsuitable or poorly suited habitat for FG1, FG2 and FG3 with high confidence; the groundwater levels of these sites were inadequate in providing the saturated conditions required by these functional groups. None of the 35 sites were deemed to have fair habitat or better for FG2, which occurs along water courses. Only two sites, 6527-589 and 6627-2374 were located within $20 \mathrm{~m}$ of a watercourse, and both these sites contained poor to fair habitat for FG2. Even when the buffer distance from watercourses was increased to $30 \mathrm{~m}$, the extra two sites, 6627-2320 and 6527-3859 had poor to fair habitat at best.
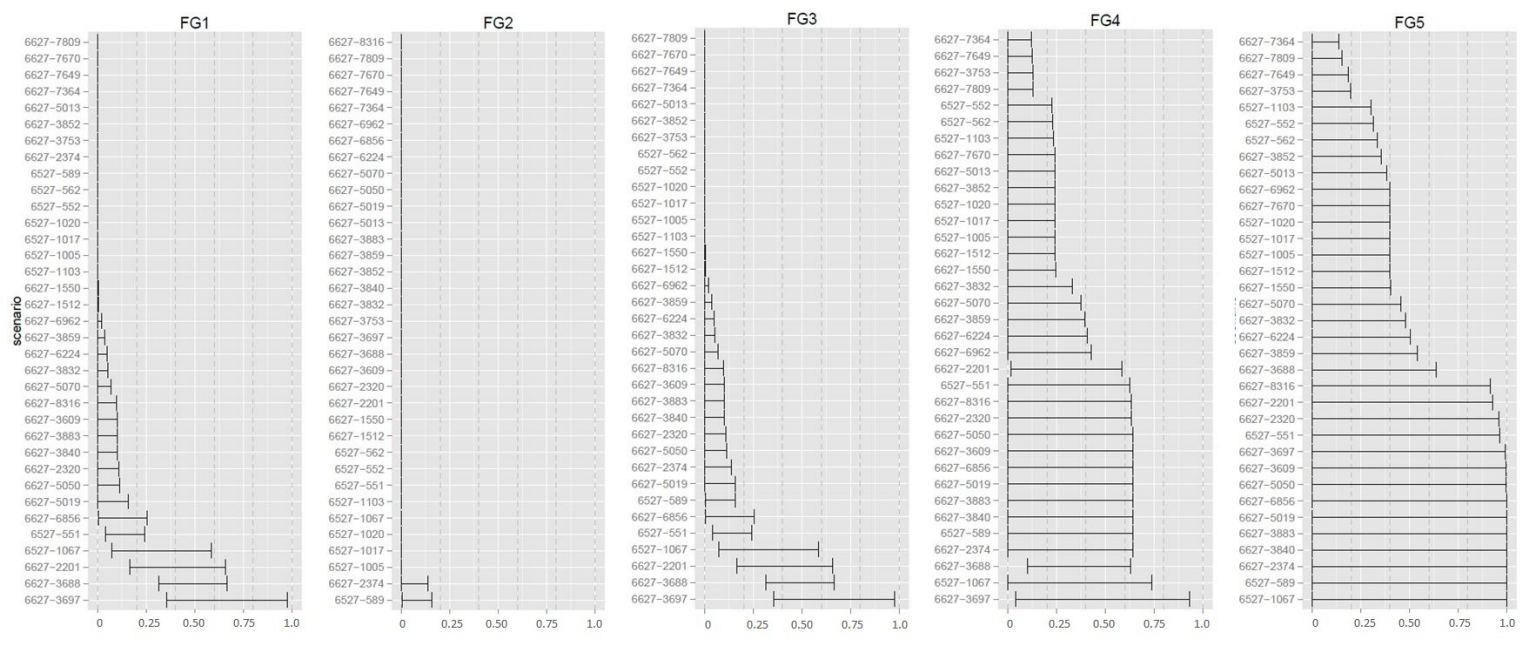

Figure 3. The habitat suitability index value bounds for the five functional groups.

FG1 and FG3 had mostly the same results, apart from the suitabilities for the sites that were within the watercourse buffer zones, which were deemed unsuitable for FG1. Under the 'best' estimate constraints, there were four sites, 6527-1067, 6627-2201, 6627-3888 and 6627-3697, that were modelled as having potentially good habitat for FG1 and FG3. Such a finding can possibly be used by land managers or researchers to identify locations where they may discover these ecological communities or, for example, where they can relocate them for conservation purposes. Site 6627-3697 may potentially have excellent habitat for these two functional groups, however it may also only have fair habitat; in other words there is uncertainty about its suitability. Although it can be said with high confidence that this site and 6627-3688 are more suitable for supporting FG1 and FG3 than most of the sites tested. 
There was clearly more uncertainty about the habitat suitabilities for FG5, which require drained soils over a shallow water table, compared to the other species (Figure 3). All sites had a minimum habitat suitability of zero for FG5. There were nine sites that had suitability bounds ranging from 0 to 100 for FG5, in other words there was complete uncertainty. FG4, which incorporated different constraints for their wetting and drying cycle, also had large uncertainty bounds, however less than FG5. For both FG4 and FG5, the sites with higher certainty were those with much deeper water tables (closer to the $20 \mathrm{~m}$ mark); for example 6627-7364 and 6627-7809 could be regarded as having unsuitable or poor habitat for these groups with high confidence. As all sites have suitability values that overlap, there is high uncertainty about the order of rank implied in the FG4 and FG5 plots.

The alternative constraints, representing different interpretations of the qualitative descriptions of the habitat suitability requirements of the functional groups, were run and found to have, at the most, relatively marginal impact on the model outputs. Three of the eight alternatives tested had no noticeable effect on the model results. Different weights for the wetting and drying cycle constraints were tested for FG4; the alternative that gave more weight to the wetting constraints slightly reduced the uncertainty bounds and the alternative that gave more weight to the drying constraints (corresponding with FG5) widened the uncertainty bounds.

The maximum and minimum habitat suitability values for each site were plotted over time. For most sites, no clear changes occurred in any of the sites over certain periods, i.e. the suitability values remained constant or fluctuated greatly or inconsistently throughout the dataset. Notable changes were apparent in some sites; examples are shown in Figure 4. At sites 6627-6856, 6527-1067 and 6627-2374, the maximum habitat suitability values for FG1, FG2 and FG3 (patterns were the same for the three groups for all sites, but only FG3 plots are shown) were potentially high during the first decade or so of the dataset and then markedly dropped after a point in time. For 6627-2374, although the maximum suitability values fluctuated year to year, it reached 1.0 at least every few years before dropping to a constant 0.1 from 1999. For site 6627-2201, the maximum habitat suitability remained high through time, however the minimum value significantly dropped around 1980. The patterns in Figure 4 indicate abrupt declines in groundwater level at the sites.

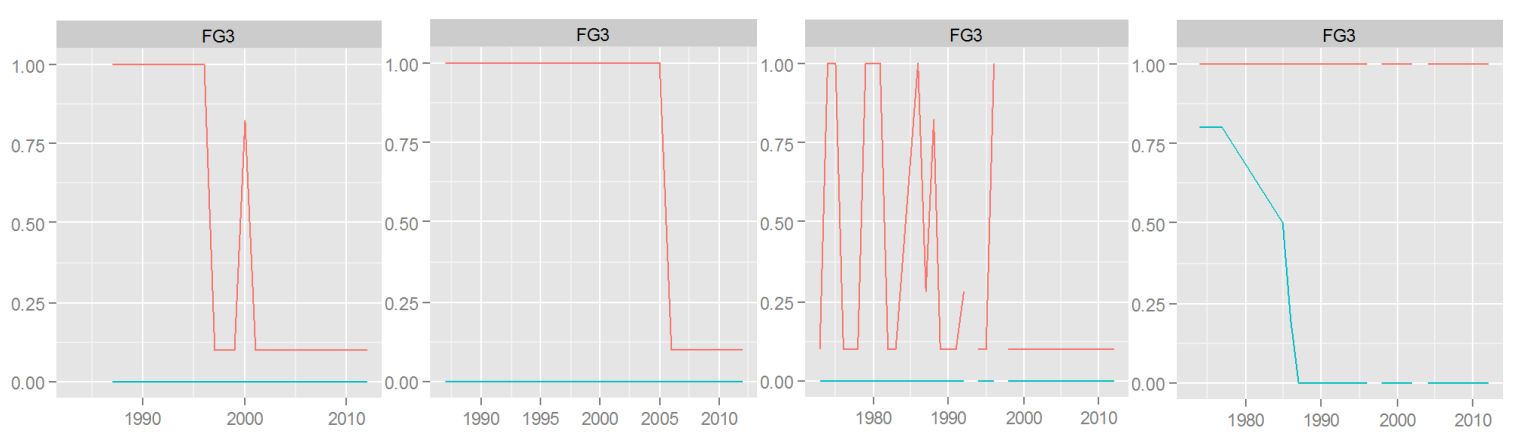

Figure 4. The maximum and minimum habitat suitability values for FG3 at sites 6627-6856, 6527-1067, 6627-2374 and 6627-2201 (left to right).

\section{DISCUSSION AND CONCLUSIONS}

This study demonstrated how limited and uncertain ecological understanding can be encapsulated in a form that allows sites to be evaluated in terms of their suitability for supporting species or species assemblages. One area of potential weakness in the approach (and most ecological modelling approaches) involves the process of articulating qualitative and often vague descriptions of the ecological system into mathematical representations. This process introduces uncertainties into the model, as this form of input is subject to biases. There were attempts to reduce these uncertainties in this study by documenting the qualitative descriptions and their translations (not presented in this paper), and testing alternative translations such as in Pollino et al. (2007). The results of the alternative translations indicated that the models were relatively robust. Another possible method of reducing uncertainties is involving multiple experts in the setting or review of model constraints.

Understanding of groundwater requirements of GDEs in the Willunga Basin is quite limited. Studies such as the one presented here may be a step towards helping to reduce some uncertainties. Future work should include incorporating ecological data into the model through the calibration or validation stage, or both. Another challenge involves extrapolating groundwater data beyond the well locations. Further work to include more attributes of the groundwater regime (e.g. timing and duration), water quality and other 
environmental factors (e.g. soil type, climate variability) into the model would increase the realism of the model and possibly its accuracy. For example, most GDE plant species utilise a combination of groundwater, surface water and water stored in soils; often opportunistically using one source more when it becomes readily accessible. Therefore a model of habitat suitability of GDEs should ideally incorporate information on the state of the other water sources as well as understanding of how the species or assemblages preferentially use the different water sources.

The model results highlighted the uncertainty about groundwater levels required by FG5, in particular. These large bounds indicate knowledge gaps that require further research. It could be that it is not possible to narrow the uncertainty bounds around the habitat suitability for these functional groups with respect to groundwater levels, due to the large variation in specific requirements of species within the groups. In which case, a narrower or different classification of functional groups may be necessary to reduce uncertainties. In addition to identifying knowledge gaps, this approach has possible management or research applications. For example it may be useful to be able to identify sites with relatively high habitat suitability to target monitoring or help locate areas for conservation. Also, through assessing the suitability values of sites through time, it is possible to identify where a significant change has potentially occurred; these sites can be targeted for further investigation, for example, to determine what activities (e.g. groundwater pumping, drainage or vegetation clearance) may have taken place and possibly caused these changes. Rather than forcing the modeller to produce precise parameter values, the approach embraces uncertainty and thereby facilitates an analysis that is more true to the state of knowledge about the ecological system. In other words the approach does not attempt to understate the uncertainty about the ecological processes the model represents.

\section{REFERENCES}

AMLRNRM (2007). Water Allocation Plan for the McLaren Vale Prescribed Wells Area. Government of South Australia. Adelaide and Mount Lofty Ranges Natural Resources Management Board.

Department for Water (2012) McLaren Valey PWA. Groundwater level and salinity status report 2011. Science, Monitoring and Information Division, Department for Water, State of South Australia.

EA and SKM (2012). Environmental Water Requirements for Groundwater Dependent Ecosystems of the Adelaide Plains and McLaren Vale. Ecological Associates Report for Adelaide and Mount Lofty Ranges Natural Resources Management Board, Eastwood.

El Sawah, S., Guillaume, J. and Mitchell, M. (2011). 'Using Participatory Rapid Appraisal and DPSIR approaches for participatory modelling: A case study for groundwater management in South Australia', International Congress on Modelling and Simulation (MODSIM 2011), ed. Felix Chan, Modelling and Simulation Society of Australia and New Zealand Inc., Australia, pp. 2873-2879.

$\mathrm{Fu}$, B. and Guillaume, J.H.A. (under review). Assessing certainty and uncertainty in habitat suitability models by comparing extreme cases. Submitted to Environmental Modelling and Software.

Knowles, I., Teubner, M., Yan, A., Rasser, P. and Lee, J.W. (2007). Inverse groundwater modelling in the Willunga Basin, South Australia. Hydrogeology Journal 15 (6), 1107-1118.

MacKay, H. (2006). Protection and management of groundwater-dependent ecosystems: emerging challenges and potential approaches for policy and management. Australian Journal of Botany 54, 231-237.

McMahon, T.E. (1983). Habitat Suitability Index Models: Coho Salmon. Fish and Wildlife Service, U.S Department of the Interior, Washington, DC. FWS/OBS-82/10.49. 29 pp.

Pollino, C. A., White, A.K. and B. T. Hart (2007). Examination of conflicts and improved strategies for the management of an endangered Eucalypt species using Bayesian networks. Ecological Modelling 201: 37-59.

Smith, C.S., Howes, A.L., Price, B. and McAlphine, C.A. (2007). Using a Bayesian belief network to predict suitable habitat of an endangered mammal - the Julia Creek Dunnart (Sminthopsis douglasi). Biological Conservation 139, 333-347. 